\title{
Un pequeño cambio en la ética del profesor universitario

\author{
A Small Change in the Ethics of the University Professor
} Uma pequena mudança na ética do professor universitário
}

\author{
Víctor Hugo Robles-Francia \\ Universidad Juárez Autónoma de Tabasco, México \\ vicrob13@yahoo.com.mx \\ https://orcid.org/0000-0003-1046-4768
}

\section{Resumen}

Este artículo reporta el efecto en el juicio moral de 36 profesores universitarios producido por una intervención basada en la discusión emocional de dilemas morales. Para ello, se asumió el juicio moral como variable dependiente, el pretest y el postest de la intervención como variable independiente y se controló el experimento mediante la covarianza de la edad. El efecto del tamaño de la intervención fue nulo a partir del valor de la eta parcial al cuadrado. Pero el cambio del juicio moral de los académicos fue positivamente pequeño en 4.41 puntos. Sin embargo, el valor correspondiente de la edad evidenció un efecto mediano en el incremento del juicio moral. De hecho, se pudo apreciar que la estructura del razonamiento moral en adultos, como los académicos estudiados, es flexible aún, y no tan inmutable como se ha reportado en otras investigaciones. En este sentido, conviene revisar los dilemas morales emocionales considerando la edad como parte del diseño del dilema.

Palabras claves: dilemas-morales, discusión-emocional, intervención, juicio-moral, profesores-universitarios. 


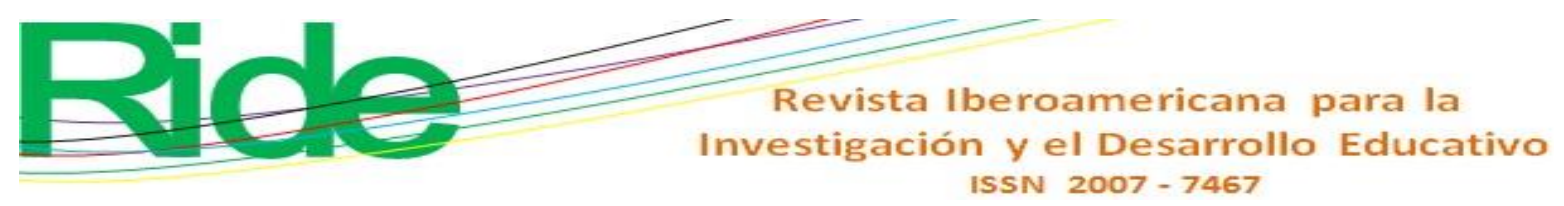

\begin{abstract}
This article reports the effect on the moral judgment of 36 university professors produced by an intervention based on the emotional discussion of moral dilemmas. For this, the moral judgment was assumed as a dependent variable, the pretest and the posttest of the intervention as an independent variable and the experiment was controlled by the covariance of age. The intervention size effect, the partial eta squared was zero. But the change in the moral judgment of the academics was positively small at 4.41 points. However, the corresponding value of age evidenced a median effect in the increase of moral judgment. In fact, it could be seen that the structure of moral reasoning in adults, like the academics studied, is still flexible, and not as immutable as has been reported in other investigations. In this sense, it is convenient to review emotional moral dilemmas considering age as part of the design of the dilemma.
\end{abstract}

Keywords: Moral-dilemmas, emotional-discussion, intervention, moral-judgment, university-professors.

\title{
Resumo
}

Este artigo relata o efeito no julgamento moral de 36 professores universitários produzido por uma intervenção baseada na discussão emocional dos dilemas morais. Para tanto, o julgamento moral foi assumido como variável dependente, o pré-teste e o pós-teste da intervenção como variável independente e o experimento foi controlado pela covariância da idade. O efeito do tamanho da intervenção foi zero do valor da eta parcial ao quadrado. Mas a mudança no julgamento moral dos acadêmicos foi positivamente pequena em 4,41 pontos. No entanto, o valor correspondente da idade evidenciou um efeito mediano no aumento do julgamento moral. De fato, pode-se ver que a estrutura do raciocínio moral em adultos, como os acadêmicos estudados, ainda é flexível, e não tão imutável quanto foi relatado em outras investigações. Nesse sentido, é conveniente rever os dilemas morais emocionais considerando a idade como parte do desenho do dilema. 


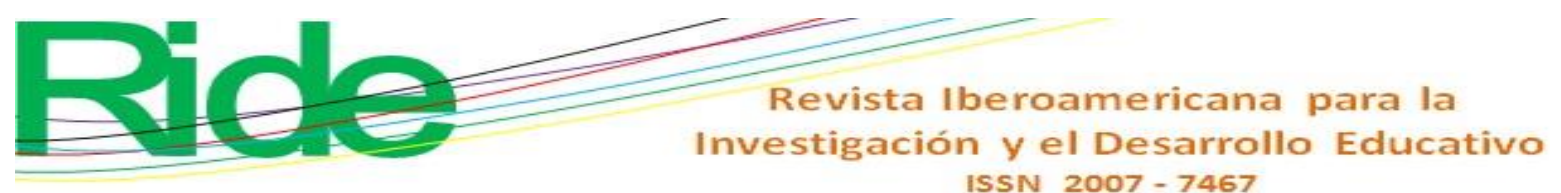

Palavras-chave: dilema-moral, discussão-emocional, intervenção, julgamento moral, professores universitários.

Fecha Recepción: Enero 2018

Fecha Aceptación: Julio 2018

\section{Introducción}

Según Boroel y Arámburo (2016), los docentes de las diversas disciplinas reconocen la importancia de la universidad como un espacio idóneo para la enseñanza y la formación ética y la moralidad, lo cual sucede porque desempeñan el rol de agente moral al continuar con la funciones restrictiva de los padres y la familia (Shelina y Mitina, 2015). Esto significa que el grado de desempeño como profesor se relaciona con el nivel del juicio moral (Cartwright y Simpson, 1990), el cual no suele ser asumido por los programas de preparación de maestros (Zagorin, 1998).

Esto, evidentemente, puede generar múltiples inconvenientes, pues si los profesores desconocen las fases de las decisiones morales, entonces estarán imposibilitados para gestar su propio cambio y para incidir favorablemente en los estudiantes (Douglas, 2006), ya que como lo expresa Rojas y Deulofeu (2015), lo que realmente impacta en sus estudiantes es la coherencia del profesor en torno a lo que dice y hace, de ahí que sea necesario el perfeccionamiento de la competencia del juicio moral del docente para enfrentar la moralidad contemporánea (Sileo, Sileo y Pierce, 2008). Esto es indispensable porque en diversos estudios se ha demostrado que un bajo nivel de competencia del juicio moral puede promover actuaciones deshonestas.

Como ejemplo de esta afirmación se puede tomar el estudio de Diez-Martínez (2015), quien en su investigación señala la actitud indiferente que suelen tomar algunos docentes frente a conductas inadecuadas como el plagio, la compra de tareas por parte de los alumnos o la falta de transparencia en las acreditaciones. Lo que sucede, como lo explican Pečujlija, Cosić y Ivanišević (2011), es que la moralidad de los profesores depende de las situaciones particulares en que se encuentren, así como de la conciencia moral para fundamentar su 
razonamiento en la toma de decisiones (Angulo y Acuña, 2005). Dicho de otra manera, el compromiso ético no pasa simplemente por el comportamiento deontológico, sino por el convencimiento y la entrega con la labor realizada, la cual consiste en la formación de mejores seres humanos (Rojas , 2011).

Por este motivo, la presente indagatoria procura ampliar las investigaciones sobre el incremento del razonamiento moral de los docentes en torno a las consecuencias y la elección entre diferentes cursos de acción mediante la deliberación emocional sobre normas individuales y colectivas, denominada en este caso como competencia del juicio moral de docentes universitarios (García, 2010), la cual se está convirtiendo en un fundamento de la enseñanza y disposición efectiva, a través del desarrollo de nuevos programas que empiezan a concebir el desarrollo moral como asignatura esencial (Johnson, 2008). Esto con el propósito de ofrecer “dotaciones adicionales” para formar un educador democrático y equitativo.

Sobre este aspecto, el trabajo de Johnson (2008) demuestra, por una parte, que el juicio moral es un constructo clave de la disposición del docente porque le permite reconocer las dimensiones multifacéticas del juicio moral de los estudiantes $\mathrm{y}$, por la otra, que el comportamiento del profesor afecta a los estudiantes en su educación moral, en la cual utilizan sus propias experiencias como dilemas morales para obtener un significado más realista.

Asimismo, sobre el juicio moral de profesores vale comentar la investigación de AlShurai (2012), quien encontró un razonamiento predominantemente convencional de la taxonomía de Kohlberg en los participantes de su estudio (100 maestros de secundaria de Kuwait cuya edad promedio era de 28.6 años). Al respecto, vale comentar que las intervenciones en el desarrollo moral considera la base cognitiva expuesta por Kohlberg (1958), quien señala que las personas llegan a la vida adulta en un estadio convencional y excepcionalmente logran un nivel poscovencional reflexivo de principios universales. Para este autor, el juicio moral se establece en un estadio o estructura cognitiva lógica del sujeto, que pondera las acciones de los otros respecto a las suyas y lo incita a equilibrar la situación 

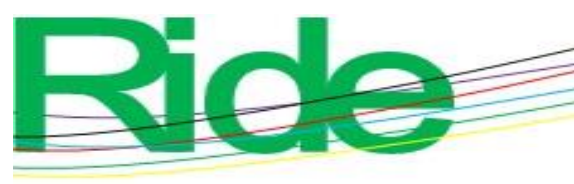

Revista Iberoamericana para la

Investigación y el Desarrollo Educativo

ISSN 2007 - 7467

en una posición justa. En tal sentido, Lind (2004) define el juicio moral como una competencia consistente que estimula al individuo a actuar.

Las intervenciones en el juicio moral asumen el precepto del estadio cognitivo, la base personal de disertación lógica sobre lo correcto o incorrecto de una acción, mediante la cual se delibera sobre el estímulo y lo impulsa a tomar una decisión sobre lo correcto de la historia o del dilema, semejando el proceso de Kolberg (1992) en los dilemas durante su entrevista. La teoría del juicio moral de Kohlberg (1958, 1964, 1992), basada a su vez en el constructo estadio de Piaget (1974), ha sustentado el trabajo desarrollado por Rest y Narváez (1998), Pérez (1997), Lind (2005), Heredia (2013), Carrillo (1992), entre otros. Ello ha marcado una época sobre la cognición de las reglas, su cumplimiento y el sentido de justicia mediante los seis estadios de juicio moral.

Sin embargo, Amorim Arantes De Araújo y Sastre Vilarrasa (2003), así como Greene y Haidt (2002) muestran desubrimientos acerca de la predominancia del pensamiento emocional en el jucio y en el crácter moral. Los señalamientos de estos autores, además de los resultados exiguos de las intervenciones basadas en la teoría cognitivista, que sustenta la discusión de dilemas morales, realizados por Gutiérres y Vivó (2005), Hernández-Mendo y Planchuelo (2014) y Robles (2011a), obligan a considerar el componente emotivo como parte importante de la intervención del juicio moral, pues dicho aspecto influye en diversos procesos intrapsíquicos e interpersonales (Tracy y Robins, 2004). A partir de esto, se conformó la siguiente hipótesis:

\section{Hipótesis}

La discusión de dilemas morales emocionales incide favorablemente en la competencia del juicio moral de los profesores. Operacionalmente, la intervención mediante la discusión de dilemas y las emociones con el literal $\mathrm{K}_{\mathrm{e}}$, el índice $\mathrm{C}$ con la $\mathrm{C}$ y el incremento con $\Delta$, se tiene:

$$
\mathrm{Si} \mathrm{K}_{\mathrm{e}} \Rightarrow \Delta \mathrm{C}
$$




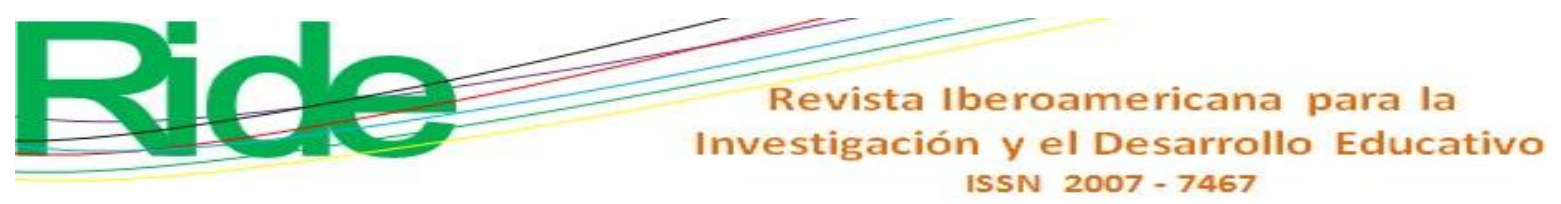

\section{Método}

En la presente investigación se realizó un experimento, en el sentido de comparar las modificaciones que una variable independiente o una acción dirigida pueden provocar en una variable dependiente, como sugiere Rodríguez (2011). En concreto, el experimento consistió en contrastar los cambios causados por el método de discusión de dilemas morales emocionales sobre el juicio moral de los profesores. Para analizar el efecto de la intervención se ejecutó la prueba univariada de varianza mediante el software estadístico SPSS (versión 22.0), y se observó el resultado del valor de la eta parcial cuadrada sobre el pretest y postest del juicio moral. El juicio moral se cuantificó mediante el índice C de Lind (2004) como variable dependiente, el test (pretest y postest) como factor fijo y la edad como covariable (López-Roldán y Fachelli, 2015).

\section{Participantes}

Los participantes fueron 36 profesores universitarios (27 con maestría y 9 con licenciatura) de un tecnológico del sureste mexicano, con una edad promedio de 36.89 años (14 femeninos y 22 masculinos) y con un promedio de 13.59 años de experiencia profesional. Asimismo, con un aprovechamiento escolar en su último grado de estudios de 9.05 sobre 10, y 19.98 años escolares cursados (Tabla 1).

Tabla 1. Datos de los participantes

\begin{tabular}{cccccc}
\hline $\begin{array}{c}\text { Edad } \\
\text { promedio }\end{array}$ & Género & $\begin{array}{c}\text { Experiencia } \\
\text { profesional }\end{array}$ & $\begin{array}{c}\text { Desempeño } \\
\text { escolar }\end{array}$ & $\begin{array}{c}\text { Grado } \\
\text { académico }\end{array}$ & $\begin{array}{c}\text { Años escolares } \\
\text { cursados }\end{array}$ \\
\hline 36.89 & $\begin{array}{c}14 \text { femeninos } \\
22 \text { masculinos }\end{array}$ & 13.59 & 9.05 & $\begin{array}{c}27 \text { maestría } \\
\text { 9 licenciatura }\end{array}$ & 18.98 \\
\hline
\end{tabular}

Fuente: Elaboración propia 


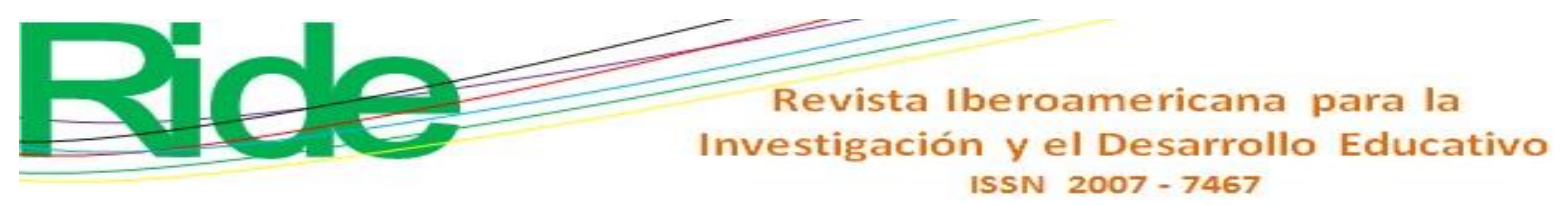

\section{Instrumento}

Todos los participantes completaron, antes y después de la intervención, el Cuestionario del Juicio Moral CJM de Lind (2008). Mediante el CJM se cuantificó el índice C del juicio moral (Lind, 1999), que mide la consistencia de razonamiento del sujeto y se centra en su calidad. El CJM tiene dos historias que forman dos respectivos dilemas, seis argumentos a favor y seis en contra de la decisión hecha por el protagonista de la historia. En esta cada argumento representa una de las seis orientaciones básicas descritas por Kohlberg (1992). Así, los ítems conforman un diseño experimental 2x2x6, contesta sobre su grado de acuerdo con la resolución del dilema y el nivel de aceptación de cada uno de los ítems correspondientes a los seis estadios kohlbergianos, en una escala diferenciada de -4 hasta +4 .

\section{Intervención}

El procedimiento aplicado siguió lo sugerido por Robles (2011b), con cinco sesiones en una semana, de 90 minutos cada una. Además, en la cuarta y quinta sesión se integró el diseño y exposición de un dilema propio de los participantes:

a. Exposición por el profesor. En cada una de las tres primeras sesiones se presentó un dilema moral emocional distinto a los sujetos participantes, los cuales fueron narrados por el facilitador. A los participantes se les estimuló para que participaran a través de preguntas que se les plantearon para intentar conocer sus sensaciones emocionales durante la presentación del dilema. Seguidamente, se les invitó a compartir sus percepciones y a reflexionar con los demás participantes en pequeños grupos, con lo cual se procuró estimular un aumento en el juicio moral (Robles, 2011b).

b. Elaboración y discusión de dilemas: En esta parte del procedimiento (cuarta y quinta sesión) los propios participantes diseñaron un dilema a partir de sus contextos disciplinares. Este fue expuesto y discutido entre todos, integrando parte del procedimiento de discusión de dilemas de Robles (2011b) y una fracción del programa de intervención de Hernández-Mendo y Planchuelo (2014). 


\section{Resultados}

El índice C, la competencia del juicio moral de los 36 profesores participantes, antes de la intervención fue de 12.94 puntos sobre 100, con una desviación media de 0.128 y una media de error estándar de 0.0213, mientras que luego de la intervención obtuvo 17.35 puntos, una desviación media de 0.104 y una media de error estándar de .0173 (Tabla 2). Esto representó un cambio favorable (4.41 puntos) con menor desviación estándar y menor media de error estándar.

Tabla 2. Estadísticas de grupo

\begin{tabular}{cccccc}
\hline & TEST & $\mathrm{N}$ & Media & $\begin{array}{c}\text { Desviación } \\
\text { estándar }\end{array}$ & $\begin{array}{c}\text { Media de error } \\
\text { estándar }\end{array}$ \\
\hline \multirow{2}{*}{ Índice C } & Pretest & 36 & 12.94 & .128 & .0213 \\
& Postest & 36 & 17.35 & .104 & .0173 \\
\hline
\end{tabular}

Fuente: Elaboración propia

Igualmente, se realizó la prueba de normalidad de Shapiro Wilk sobre la diferencia del índice C, pretest y postest, que aportó un valor de 0.268 mayor a 0.05 (Tabla 3), con lo cual se rechaza la hipótesis nula, pues se demuestra que se tiene una distribución normal.

Tabla 3. Prueba de normalidad de la diferencia pretest y postest

Shapiro-Wilk

Estadístico Gl Sig.

\begin{tabular}{cccc} 
& Estadistico & Gl & \multicolumn{2}{c}{ Sig. } \\
\hline Diferencia pre-pos-test & .963 & 36 & .268 \\
\hline
\end{tabular}

Fuente: Elaboración propia

\section{Análisis estadístico y prueba de hipótesis}

El análisis se realizó de la siguiente forma: en función del tamaño de los grupos y de la condición de normalidad, se aplicó la prueba paramétrica del análisis de varianza que tiene una distribución normal, como lo sugiere Gómez-Gómez, Danglot-Banck y Vega-Franco (2013). Para observar el resultado de la intervención se realizó el análisis univariado de varianza, particularmente observando el tamaño del efecto a través de la eta parcial cuadrada. Para ello, se estableció el índice C como variable dependiente, como variable independiente 
(factor fijo) el test (pretest y postest) y como variable de control (covariable) la edad (LópezRoldán y Fachelli, 2015).

La hipótesis fue parcialmente soportada. La intervención mediante la discusión de dilemas morales emocionales incidió favorablemente en la Competencia del Juicio Moral de los Profesores CJMP. La CJMP, cuantificada mediante el índice C, tuvo un cambio positivo de 4.41 sobre 100, mientras que en el cambio del juicio moral se observó menor desviación estándar y menor media de error estándar (Fig. 1). Sin embargo, se aprecia que el efecto del tamaño, a partir del valor de la eta parcial al cuadrado del test (pretest y postest) de 0.004, fue nulo en el índice C. Contrariamente, el valor de la eta parcial cuadrada de la edad registró un valor de 0.125 , evidenciando un efecto mediano de la edad en el incremento del índice $\mathrm{C}$ (Tabla 4). Asimismo, la prueba de efectos intersujetos registró un valor $p$ de 0.009 , menor que 0.05 , un modelo corregido significativo del índice $\mathrm{C}$ a través de las variables test y edad, donde la variable test (pretest y postest) tuvo un valor $p$ de 0.604 , mayor que 0.05 , mostrando que no incide en el índice $C$; el valor $p$ de la edad 0.003 menor que 0.05 sí explica el índice C (Tabla 4).

Tabla 4. Prueba de efecto inter-sujetos

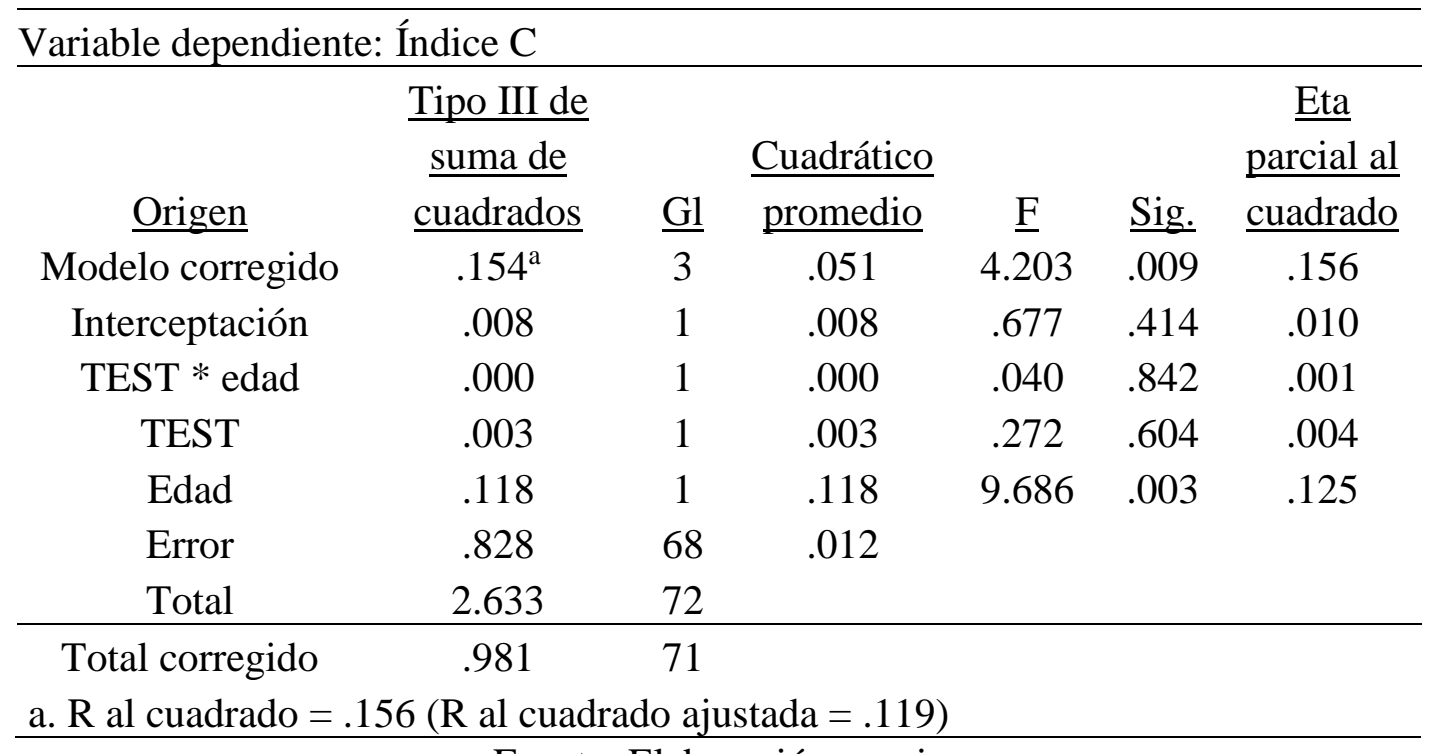

Fuente: Elaboración propia 

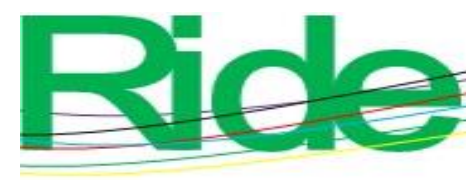

Revista Iberoamericana para la Investigación y el Desarrollo Educativo ISSN $2007-7467$

Figura 1. Resultados promedios del índice $\mathrm{C}$ producidos por la intervención

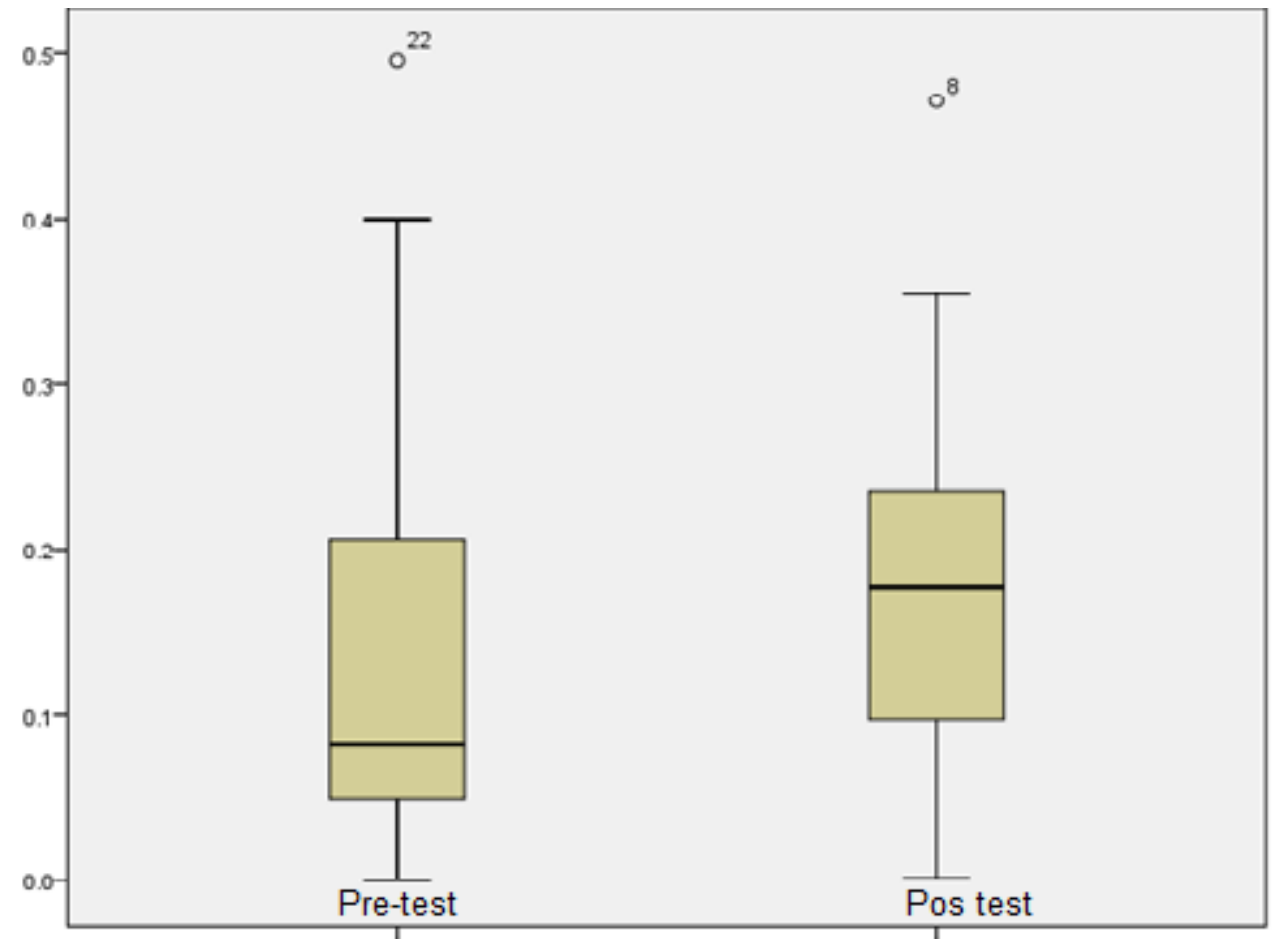

Fuente: Elaboración propia

\section{Discusión}

El aumento de 4.41 puntos, con menor desviación estándar y menor media de error estándar, de la competencia del juicio moral de los profesores de 36.89 años, después de la intervención mediante la discusión de dilemas morales emocionales, no representó efecto alguno. Esto es semejante a lo encontrado por Gutiérrez y Vivó (2005), quienes no reportaron cambios significativos en el grupo experimental intervenido de adolescentes de nivel secundaria, aunque sí registraron algún aumento promedio. Por otro lado, resultados previos de Robles (2011a) sobre la competencia del juicio moral en adultos habían tenido resultados negativos. En este sentido, es valioso el ligero incremento en la adultez estudiada.

El cambio positivo de la competencia del juicio moral de los profesores en la presente investigación se asemeja a lo encontrado por Hernández-Mendo y Planchuelo (2014), aunque en niños de 8 a 11 años, cuyo nivel de juicio moral tuvo un cambio pequeño de preconvencional a convencional. El procedimiento de intervención en el presente estudio 

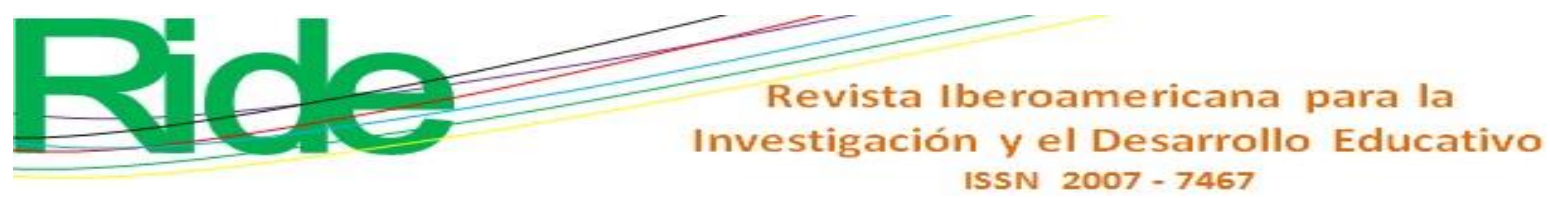

integró una etapa donde los participantes diseñaron y expusieron su propio dilema moral emocional, semejante a la etapa donde Gutiérrez y Vivó (2005) hicieron que los adolescentes realizaran, expusieran y discutieran un dilema moral. De esto se rescata, conforme a los resultados de Gutiérrez y Vivó (2005) y Hernández-Mendo y Planchuelo (2014), la discusión de dilemas, y en virtud de los resultados propios de esta investigación, las emociones en el diseño y discusión de los dilemas morales.

Asimismo, y aunque no fue parte de la hipótesis de esta investigación (solo fue una variable de control), la edad sí tuvo un efecto mediano en la competencia del juicio moral. De hecho, se pudo apreciar que la estructura del razonamiento moral en la edad adulta de los académicos estudiados es maleable aún, y no tan sólida e inquebrantable, como lo señala Kohlberg (1992) y como lo puntualiza Pérez (1997). En este sentido, conviene revisar los dilemas morales emocionales considerando la edad como parte del diseño del dilema, así como el procedimiento para ajustarlo a las correspondientes edades de la discusión del conflicto planteado.

\section{Conclusiones}

El pequeño aumento en el juicio moral de los profesores es un aliciente para ahondar en la búsqueda de su perfeccionamiento en la adultez a través de intervenciones basadas en emociones en los dilemas morales. Asimismo, un proceso proactivo donde los participantes expongan, discutan y creen dilemas tomando como base sus propios contextos facilita las condiciones para el desarrollo de juicio moral, como parte de una intervención.

Complementariamente, y a pesar de que el incremento de la competencia del juicio moral no fue un efecto propio de la intervención, la edad representó un efecto mediano en tal competencia, pues se percibió que la estructura del razonamiento moral en la edad adulta (específicamente de 36.89 años promedio en esta investigación) es elástica y no tan inmutable, como se estipula en la teoría cognitivista del juicio moral.

En la intervención realizada, por último, se compararon los cambios producidos en el índice $\mathrm{C}$, abordando los cambios producidos por una situación artificial, en este caso el efecto del método de discusión de dilemas morales emocionales sobre la competencia del juicio 
moral. Este estudio es un esfuerzo inicial sobre la afectación de las emociones en los dilemas y su intervención en el juicio moral en una población adulta, esto con el propósito de aportar una solución sobre la necesidad de desarrollar competencias clave, así como el perfeccionamiento de habilidades de la discusión para darle un cambio en la enseñanzaaprendizaje, en este caso, de la competencia del juicio moral de los académicos.

\section{Referencias}

Al-Shurai, S. (2012). An empirical investigation of the moral judgment development of a sample of high school Kuwaiti teachers. Education, 133(2), 340-348.

Amorim Arantes De Araújo, V. y Sastre Vilarrasa, G. (2003). Moralidad, sentimientos y educación. Educar (31), 47-66. Doi: doi:https://doi.org/10.5565/rev/educar.304.

Angulo, N. y Acuña, I. (2005). Ética del docente. Revista Educación en Valores, 1(3).

Boroel, B. y Arámburo, V. (2016). El posicionamiento del docente ante la formación en valores en la educación superior. Revista Iberoamericana para la Investigación y el Desarrollo Educativo, 7(13), 463-482.

Carrillo, I. (1992). Comunicación, lenguaje y educación: discusión de dilemas morales y desarrollo progresivo del juicio moral. Comunicación, Lenguaje y Educación, 4(15), 56-62. Doi: https://doi.org/10.1080/02147033.1992.1082103

Cartwright, C. and Simpson, T. (1990). The Relationship of Moral Judgment Development and Teaching Effectiveness of Student Teachers. Education, 3(1), 139-144.

Diez-Martínez, E. (2015). Deshonestidad académica de alumnos y profesores. Su contribución en la desvinculación moral y corrupción social. Sinéctica, (44), 1-17.

Douglas, I. (2006). Ética en la formación docente. Laurus, 12(21), 9-22.

García, E. (2010). Competencias éticas del profesor y calidad de la educación. REIFPO, 13(4), 29-41. Recuperado de https://eprints.ucm.es/31411/1/Competencias\%20eticas\%20del\%20profesor\%20REI FOP.pdf. 
Gómez-Gómez, M., Danglot-Banck, C. y Vega-Franco, L. (2013). Cómo seleccionar una prueba estadística. Revista Mexicana de Pediatría, 80(1), 30-34.

Greene, J. and Haidt, J. (2002). How (and where) does moral judgment work? Trends in Cognitive Sciences, 6(12), 517-523.

Gutiérrez, M. y Vivó, P. (2005). Enseñando razonamiento moral en las clases de educación física escolar. Motricidad European Journal of Human Movement, (14), 1-22.

Heredia, Y. (2013). Los dilemas morales como estrategia de enseñanza- aprendizaje para el desarrollo moral en los alumnos de preescolar. Revista de Investigación Educativa de la Escuela de Graduados en Educación, 4(7), 61-69. Recuperado de http://catedra.ruv.itesm.mx/handle/987654321/792.

Hernández-Mendo, A. y Planchuelo, L. (2014). El incremento del desarrollo moral en las clases de educación física. Revista Iberoamericana de Psicología del Ejercicio y el Deporte, 9(2), 369-392.

Johnson, L. (2008). Teacher candidate disposition: moral judgment or regurgitation? Journal of Moral Education, 37(4), 429-444.

Kohlberg, L. (1958). The development of modes of moral thinking and choice in the years ten to sixteen (Tesis doctoral inédita). Chicago, Estados Unidos: Universidad de Chicago.

Kohlberg, L. (1964). Development of moral character and moral ideology. In Hoffman, M. L. Hoffman, L. W., Hoffman, M. L. and Hoffman, L. W. (eds.), Review of Child Development Research (vol. I) (pp. 381-431). New York: Russel Sage Foundation.

Kohlberg, L. (1992). Psicología del desarrollo moral. Bilbao, España: Desclée de Brouwer.

Lind, G. (1999). Una Introducción al Test de Juico Moral (MJT). Konstanz, Alemania: Georg Lind.

Lind, G. (2004). The meaning and measurement of moral judgment competence revisited A dual-aspect model. In Fasko, D. and Willis, W. (eds.), Contemporary Philosophical and Psychological Perspectives on Moral Development and Education (pp. 185-220). Cresskill NJ, Estados Unidos: Hampton Press. 
Lind, G. (2005). Moral Dilemma Discussion Revisited -The Konstanz Method. Europe's $\begin{array}{lllll}\text { Journal of } & \text { Psychology, } & \text { Retrieved } & \text { from }\end{array}$ https://ejop.psychopen.eu/article/view/345/html.

Lind, G. (2008). The Moral Judgment Test. Retrieved from http://www.uni-konstanz.de/agmoral/mut/mjt-certification.htm\#certified_versions.

López-Roldán, P. y Fachelli, S. (2015). Metodología de la investigación social cuantitativa. Barcelona, España: Universidad Autónoma de Barcelona.

Pečujlija, M., Cosić, I. and Ivanišević, V. (2011). A Professor's Moral Thinking at the Abstract Level Versus The Professor's Moral Thinking in the Real Life Situation (Consistency Problem). Science and Engineering Ethics, 17(2), 299-320. Doi: 10.1007/s11948-009-91-x.

Pérez, E. (1997). Cognición y afecto en el desarrollo moral. Valencia, España: Promolibro. Piaget, J. (1974). El criterio moral del niño. Barcelona, España: Fontanella.

Rest, J. and Narváez, D. (1998). Defining Issues Test DIT-2 Version 3.0. Minnesota, Estados Unidos: Universidad de Minnesota.

Rodríguez, N. (2011). Diseños experimentales en educación. Revista de Pedagogía, 32(91), 147-148.

Rojas, C. (2011). Ética profesional docente: un compromiso pedagógico humanístico. Revista Humanidades, 1, 1-22.

Rojas, F. y Deulofeu, J. (2015). El formador de profesores de matemática: un análisis de las percepciones de sus prácticas instruccionales desde la "tensión" estudiante-formador. Enseñanza de las Ciencias, 33(1), 47-61. Doi: org/10.5565/rev/ensciencias.1322.

Robles, V. (2011a). La ineficacia de la discusión de dilemas morales en el crecimiento de la competencia moral en trabajadores y estudiantes. Dos casos mexicanos. Revista CES Psicología, 4(2), 47-59.

Robles, V. (2011b). Perfeccionamiento de la deliberación moral en la organización. Ciudad de México, México: Plaza y Valdés Editores.

Shelina, S. y Mitina, O. (2015). El juicio moral de profesores universitarios: un experimento multifactorial entre dos grupos. Russian Education \& Society, 57(8), 696-711. 
Sileo, N., Sileo, T. and Pierce, T. (2008). Ethical Issues in General and Special Education Teacher Preparation: An Interface with Rural Education. Rural Special Ecucation Quarterley, 27(1/2), 43-54.

Tracy, J. and Robins, R. (2004). Research Report Show Your Pride Evidence for a Discrete Emotion Expression. Psychological Science (Wiley-Blackwell), 15(3), 194-197.

Zagorin, P. (1998). Lord Acton's Ordeal: The Historian and Moral Judgment. Virginia. Quarterly Review, 74(1), 1-17. 